\title{
Research on Hysteretic Behavior of T-Type RHS Joints with Inner \\ Stiffened Plate
}

\author{
Peng Deng ${ }^{* 1, a}$, Yongpeng $\mathrm{Li}^{2, \mathrm{~b}}$,Xiaotong Shang ${ }^{2, \mathrm{c}}$, Huiling Wang ${ }^{2, \mathrm{~d}}$ \\ ${ }^{1}$ Shandong Provincal Key Laboratory of Civil Engineering Disaster Prevention and Mitigation, \\ Shandong University of Science and Technology, Qingdao, Shandong, 266590, China \\ ${ }^{2}$ College of Civil Engineering and Architecture, Shandong University of Science and Technology \\ Qingdao, Shandong 266590, China \\ adengpeng1226@163.com, b602981908 @qq.com , c173296033@qq.com, ddrawingfish@qq.com
}

Keywords: T-Type RHS joints, inner stiffened plate, hysteretic behavior

Abstract: The inner plate can be used to stiffen square tubular T-joint in order to improve the performance of square tubular $\mathrm{T}$-joint and improve the radial stiffness of square tube. The research based on numerical simulation in the paper proved that RHS joints with inner stiffened plate had better ductility, energy dissipation and seismic performance because of the change of failure mode of specimen. The effects of inner plate and hole size on hysteretic behaviour of stiffened joint have been analyzed in this artical, and the suggestion about the design of T-type RHS joints with inner stiffened plate is put forward.

\section{Introduction}

The wall of the chord in the square tubular T-joint tends to plastic deformation because the branch member generally carry axial loading [1]. Joint with Inner Stiffened Plate have been researched extensively all over the world because this joint not only can improve the radial stiffness of the chord and the ultimate capacity of the joint effectively, but also the stiffened plate located at the interior of the chord has no effect on the appearance of the structure. But researches of hysteretic performance of this joint under cyclic loading are limit [2-4]. The research by numerical simulation in the paper analyzed the effect of the inner plate and the size of hole on hysteretic behaviour of stiffened joint and put forward the suggestion about the design of this type of joint.

\section{The finite element simulated results of square tubular $\mathbf{T}$-joint}

In this paper, ten specimens are divided into three groups to study the effect of the inner plate size on the hysteretic behaviour of stiffened joint. The length of the chord and branch with Q235B are $2208 \mathrm{~mm}$ and $400 \mathrm{~mm}$. The geometric parameters of specimen are shown in Fig. 1 and Table 1.
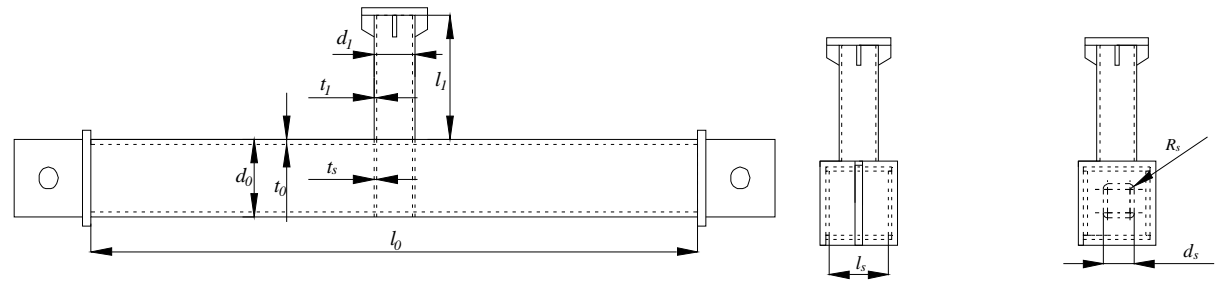

Fig.1 The geometric parameter of specimen 
Table 1 Geometric parameters of T-type RHS joint ( Unit: $\mathrm{mm}$ )

\begin{tabular}{|c|c|c|c|c|c|c|c|c|c|c|c|c|c|}
\hline specimen & $\mathrm{d}_{0}$ & $\mathrm{t}_{0}$ & $\mathrm{~d}_{1}$ & $\mathrm{t}_{1}$ & $\mathrm{Rs}$ & $\mathrm{t}_{\mathrm{s}}$ & specimen & $\mathbf{d}_{\mathbf{0}}$ & $\mathbf{t}_{\mathbf{0}}$ & $\mathbf{d}_{\mathbf{1}}$ & $\mathbf{t}_{\mathbf{1}}$ & $\mathbf{R s}$ & $\mathbf{t}_{\mathbf{s}}$ \\
\hline $\mathrm{T} 1-1-0$ & 250 & 8 & 150 & 6 & 0 & 0 & $\mathrm{~T} 1-2-2$ & 250 & 8 & 150 & 6 & 146 & 6 \\
\hline $\mathrm{T} 1-1-1$ & 250 & 8 & 150 & 6 & 118 & 4 & $\mathrm{~T} 1-2-3$ & 250 & 8 & 150 & 6 & 174 & 6 \\
\hline $\mathrm{T} 1-1-2$ & 250 & 8 & 150 & 6 & 146 & 4 & $\mathrm{~T} 1-3-1$ & 250 & 8 & 150 & 6 & 118 & 8 \\
\hline $\mathrm{T} 1-1-3$ & 250 & 8 & 150 & 6 & 174 & 4 & $\mathrm{~T} 1-3-2$ & 250 & 8 & 150 & 6 & 146 & 8 \\
\hline $\mathrm{T} 1-2-1$ & 250 & 8 & 150 & 6 & 118 & 6 & $\mathrm{~T} 1-3-3$ & 250 & 8 & 150 & 6 & 174 & 8 \\
\hline
\end{tabular}

Simulated Result of T1-1. The deformation of T1-1 under axial cyclic loading is shown in Fig.2. As shown in Fig.2 (a), T1-1-0 without inner stiffened plate appears buckling only at intersection between chord and branch member. And as shown in the Fig.2 ( b ), the inner stiffened plates and web plates between inner plates of T1-1-1 T1-1-3 appear buckling, and the lower flange of the chord between inner plates appear sinking. Deformation of joints is in the same position, and the local distortion is more obvious when the size of hole is bigger.

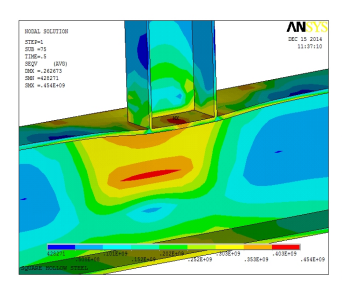

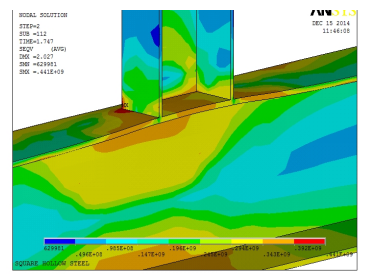

(a) $\mathrm{T} 1-1-0$
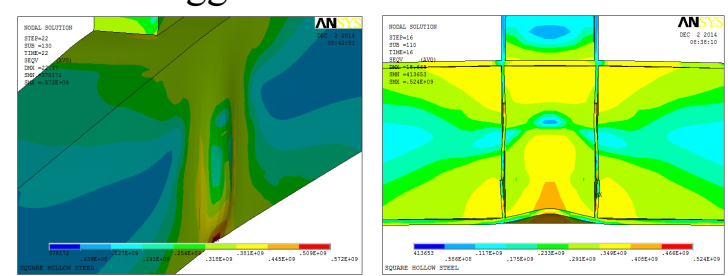

( b ) T1-1-1 T1-1-3

Fig.2 deformation of group T1-1

Simulated Result of T1-2. As shown in Fig.3 (a), the top flange of the chord near the intersection between chord and branch of T1-2-1under axial pressure appear sinking, and bottom flange of it under axial tension appear sinking. As shown in Fig. 3 (b), the deformation of T1-2-2 subjected to tension is the same as T1-2-1, but it appears buckling near the intersection between chord and branch. The deformation of T1-2-3 is similar to T1-1, but its local deformation is much less than T1-1-3 because of the shortage of radial stiffness caused by the bigger hole size.
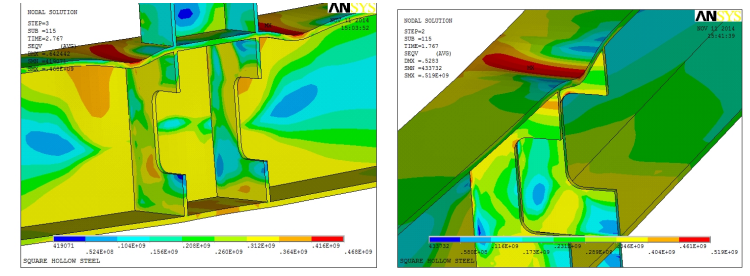

( a ) T1-2-1

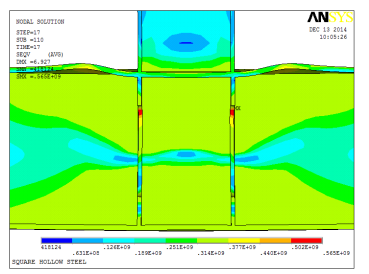

( b ) $\mathrm{T} 1-2-2$

Fig.3 Deformation of group T1-2

Simulated Result of T1-3. As shown in Fig.4 ( a ) , the deformation of T1-3-1 is similar to T1-3-2, the top flange of the chord near the intersection appear sinking under axial pressure, and the bottom flange of the chord outside the inner plates appear sinking, which is different from other specimens. This failure mode was caused by on the bigger stiffness of plate which led to the deformation of the bottom flange of chord. As shown in Fig.4 ( b ), the deformation of T1-3-3 is similar to T1-1 series, and the difference is the bottom flange of the chord between the inner plates appears buckling. 

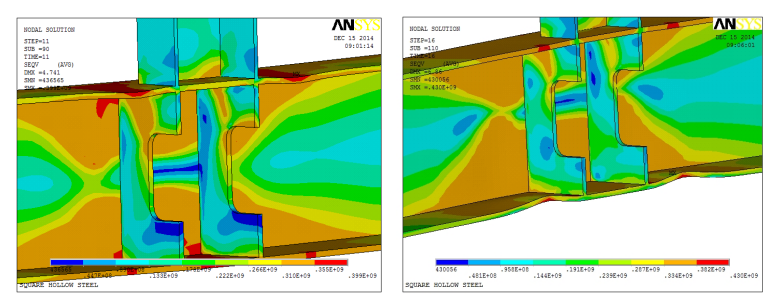

( a ) T1-3-1and T1-3-2

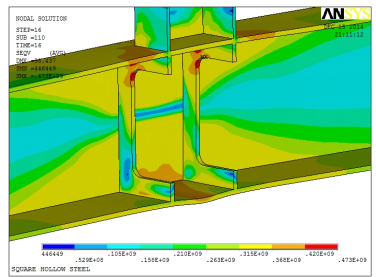

( b ) $\mathrm{T} 1-3-3$

Fig.4 Deformation of group T1-3

\section{Simulated Result Analysis}

Hysteresis Curves. As shown in Fig.5, hysteresis curves of unstiffened specimen appear pinch phenomenon obviously, while those of the joints with inner plate are full. The loop area of T1-1-1 with $47246 \mathrm{~mm}^{2}$ is the maximum, T1-1-2 with $45718 \mathrm{~mm}^{2}$ is the second, and T1-1-3 with 34051 $\mathrm{mm}^{2}$ is minimum.

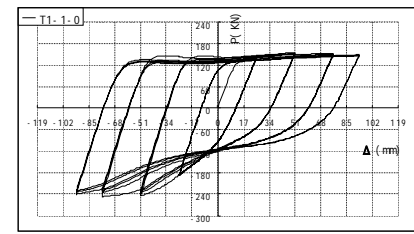

( a ) $\mathrm{T} 1-1-0$

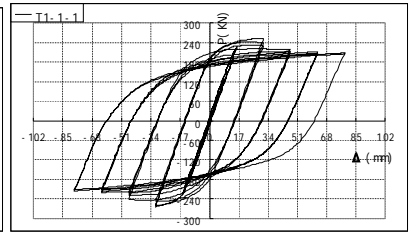

( b ) $\mathrm{T} 1-1-1$

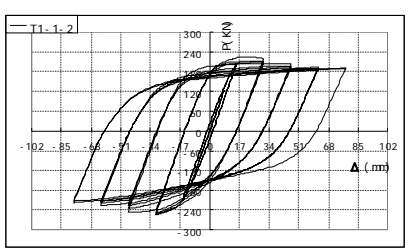

( c ) $\mathrm{T} 1-1-2$

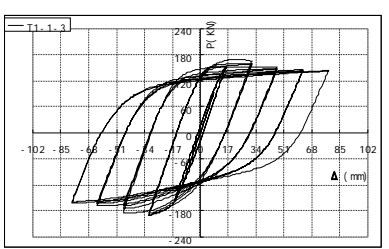

(d ) T1-1-3

Fig.5 Hysteresis curves of group T1-1

As shown in Fig.6, the carrying capacity of T-Type RHS joints with inner plate in group T1-2 whose hysteresis curves are full improved obviously. The loop area of T1-2-3 with $60892 \mathrm{~mm}^{2}$ is the maximum, T1-2-2 with $51344 \mathrm{~mm}^{2}$ is the second, and T1-1-1 with $48969 \mathrm{~mm}^{2}$ is minimum.

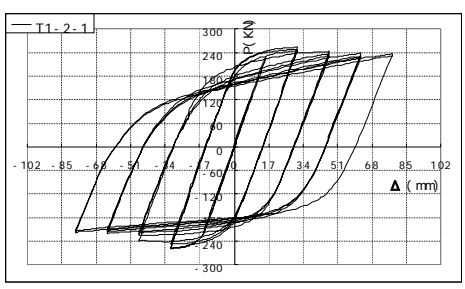

( a ) T1-2-1

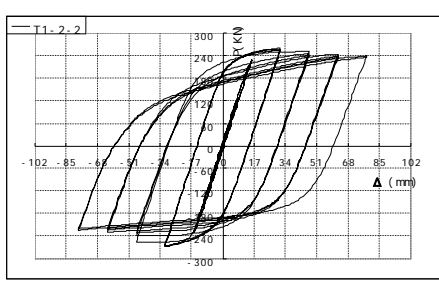

( b ) T1-2-2

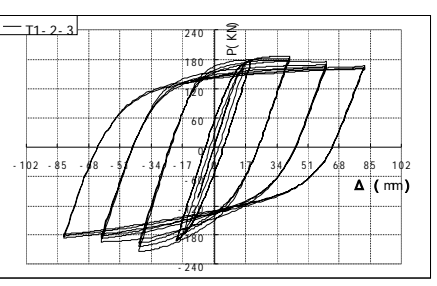

( c ) T1-2-3

Fig.6 Hysteresis curves of group T1-2

As shown in Fig.7, the hysteresis curves of the joints in group T1-3 are full. The loop area of T1-3-2 with $52591 \mathrm{~mm}^{2}$ is the maximum, T1-3-1 with $52332 \mathrm{~mm}^{2}$ is the second, and T1-3-3 with $39384 \mathrm{~mm}^{2}$ is minimum.

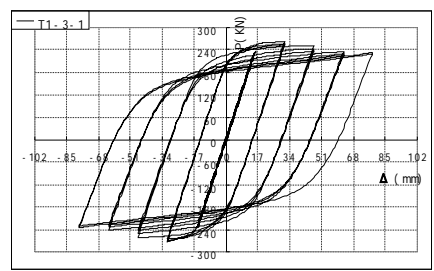

( a ) T1-3-1

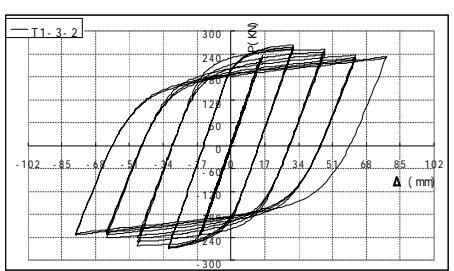

( b ) T1-3-2

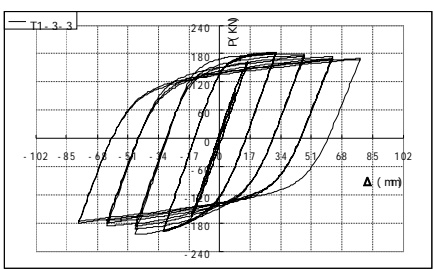

( c ) $\mathrm{T} 1-3-3$

Fig. 7 Hysteresis curve of group T1-3 
From the loop area, the effect of the size of hole on hysteretic behavior depends on the thickness of the inner plate. The recommended opening rate is about $55 \%$ in consideration of the strengthen effect and material utilization. The influence of weld has not been considered in the numerical simulation. Large welding residual stress at intersection between chord and branch can cause fatigue failure under cyclic loading. The big size of hole will lead to the insufficiencies of radial stiffness of the joint when the failure mode of specimens is the same as unstiffened specimens

Skeleton Curves. As shown in Fig.8, the elastic stage of each specimen is longer and the yield load is bigger, but there exist differences. From the skeleton curves of group T1-1, the curves decline significantly once specimens yield, which shows obvious stiffness degradation and poor ductility. Skeleton curve of T1-1-1 decline most obviously because the smaller hole size leads to the bigger stiffness of joint and the significantly stiffness of specimens. From curves of group T1-2, the stiffness degradation of specimens is not obvious, and there is no degradation in compression sections. The curves of T1-2-3 are smooth in tension sections which show better ductility and the most ideal stiffened effect. From curves of group T1-3, two skeleton curves but T1-3-3 decline significantly, which shows that the ductility of T1-3-3 is better.

Compared with the curves of T1-2-3 and T1-3-3, they are flat relatively after pecimens yield, which shows the effect of energy dissipation is ideal. Combined with hysteresis loop area, the inner plate thickness of $\mathrm{T} 1-2-3$ is the prior choice and opening rate is about 55\%, when energy-dissipating ability of specimen is better.

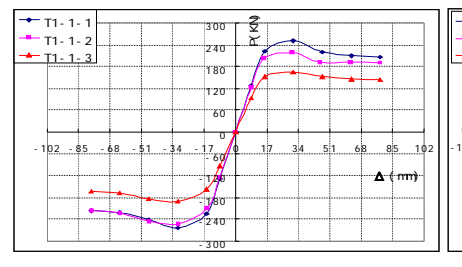

( a ) T1-1

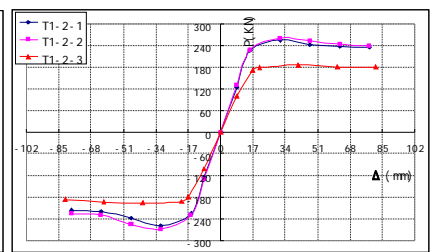

( b ) T1-2

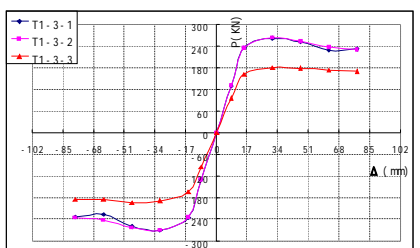

( c ) T1-3

Fig. 8 Skeleton curve of group T1

\section{Conclusions}

Although different inner plates cause different sinking and buckling, they all increase the overall deformation of the specimens, which enhances the absorb capacity and dissipate seismic energy of specimens and improves the hysteretic behavior of joints. The effect of the hole size on hysteretic behavior depends on the inner plate thickness and recommended opening rate is about $55 \%$ in consideration of the strengthen effect and material utilization. The improvement of the joint stiffness and the hysteretic behavior is limited if the plate thickness is too small, and the joint has big stiffness if the inner plate is too thick which will lead to specimen damage before the inner plate yields and low utilization rate of material, so the plate thickness is suggested to take the branch wall thickness.

\section{References}

[1] Redwood, R. G. The behavior of joints between RHS members[J]. Civil Engineers and Public Works Review, 1966

[2] Eastwood, W., Wood, A. A. Welded joints intubular structures involving rectangular resctions [J]. Univ. of Sheffileld, England, 1970: Section A Paper2

[3] Gibstein, M. B. The static strength of T-joints subjected to in-plane bending [J]. Det Norske 
Veritas Report, 1976: 76-137

[4] Wardenier J. Hollow section Joints [M]. Delft University Press, 1982 